\title{
RM42
}

\section{Permeability and SW Modeling In Carbonate Reservoirs Using Cloud Transform}

\section{ABDEL GHANI GUEDDOUD (ADMA-OPCO) \& M. Essam* (ROXAR)}

\section{SUMMARY}

The Cloud transform reproduces the conditional distributions of a dependent 3D parameter given an independent 3D parameter (Bashore et al. 1994). This distribution is estimated empirically from well data and the secondary variable. The estimated distribution will then be the basis for the cloud transform. This works in the same way as the Normal score transform except that the CDF being used is a 2D CDF estimated empirically from well data and the independent 3D parameter. This allows specifying a model that reproduces the scatter plot from the wells in a $3 \mathrm{D}$ volume.

In a case study from offshore Abu Dhabi, the relationship between porosity and permeability is found to be non-linear and Cloud transform technique was applied for permeability distribution. The porosity, which is populated using Gaussian Simulation used as independent 3D parameter and an empirical relationship was derived between porosity (3D parameter) and log derived permeability (calibrated to core at well location for each rock type, which was used to estimate permeability distribution for the Cloud transform. The initial simulation results show positive results where good history match was reached without applying multiplier for the producing wells. 


\section{Permeability and SW Modeling in Carbonate Reservoirs Using Cloud Transform}

Mohamed Essam* (ROXAR-Emerson, Abu Dhabi, UAE), Abdel Ghani Gueddoud (ADMA-OPCO, Abu Dhabi, UAE).

\section{ABSTRACT:}

Permeability distribution in a geo cellular model is always a challenge particularly in carbonate reservoirs where the relationship between porosity and permeability is nonlinear. In such cases, the traditional methodology of co-simulation or linear regression between porosity and permeability does not provide a satisfactory permeability distribution.

\section{StUdy AREA:}

The area of study is located in offshore Abu Dhabi and The Reservoir is Sabkha Cycles, Supratidal/ Intertidal mainly Dolomites and Anhydrites, subordinates of Limestone.

\section{EXECUTIVE SUMMARY:}

A 3D Geocellular Static reservoir model has been constructed over upper Jurassic reservoir using Roxar software IRAP RMS. petrophysical model is constrained by the Rock type model. A new approach was introduced to model the permeability and the water saturation by using the cloud transform algorithm.

Dynamic simulation was done to validate the modeling results.

\section{INTRODUCTION:}

In this case study we used the Cloud transform which is simply the method of reproducing the conditional distributions of a dependent 3D parameter given an independent 3D parameter (Bashore et al. 1994). This distribution is estimated empirically from well data and the secondary variable. The estimated distribution will then be the basis for the cloud transform. The CDF being used is a 2D CDF estimated empirically from well data and the independent 3D parameter. This allows specifying a model that reproduces exactly the same scatter plot from the well data in a 3D Parameter.

In this case study from offshore Abu Dhabi and as a fact at all the carbonate reservoirs, the relationship between porosity and permeability used to be found non-linear hence Cloud transform technique was applied for permeability distribution.

The porosity was populated first and then used as an input (independent 3D parameter) to model the permeability. An empirical relationship was derived between porosity (3D parameter) and log derived permeability (calibrated to core at well location) for each rock type, which the estimator used to estimate the permeability distribution for the Cloud transform. 
The Dynamic simulation results show positive results where fabulous history match was reached without applying any permeability multipliers for the producing wells.

\section{Cloud Transform Overview:}

The porosity $\mathrm{PHI}$ axis is split into $\mathrm{N}$ classes (bins) as for a simple histogram, Then for each PHI-class, calculate the corresponding $\mathrm{K}$ distribution, Then Define the K-distributions by its cumulative density function (CDF). Once the $\mathrm{K}$ distributions for each PHI class have been defined, that's it!

To transform a 3D porosity parameter look for each porosity sample in which PHI class it lies and sample from its $\mathrm{K}$ distribution (Figure.1).

\section{Porosity Modeling Summary}

After the Rock Types modeling, Porosity was distributed stochastically with a Variogram model and simple data transformation sequence (data truncation, mean, and compaction trend) conditioned to the RRT Model. (Figure.2)

All the statistical QC steps were done to validate the porosity model.

\section{Permeability Modeling Workflow Using Cloud Transform:}

Non linear transformation of the modeled Porosity based on the PHI-K cloud (scatter plot) by using the non-linear relationship between PHI and $\mathrm{K}$ and its variability. (Figure.3)

\section{Permeability Model Qc:}

Scatter plot of modeled PERM vs. BWs shows that the model can reproduce the same scattered plot of the original log data. (Figure.4)

\section{Permeability Model Validation By DYNAMIC HISTORY MATCH:}

The Dynamic simulation results show positive results where good history match was reached without applying any permeability multipliers for the producing wells. (Figure.5)

\section{Water Saturation Modeling}

SW was modeled using the same approach of cloud transform.

In this case we create a Height parameter and then transforming that to SW using a non linier transform/regression.

(Figure.6)

\section{WATER SATURATION MOdelQc:}

As a QC step a scatter plot between modeled SW vs. BWs SW (Green Dots) was done to check if the model reproduced the same scatter plot of SW 
and capture the overall heterogeneity in the reservoir or not .

As we can see we was able to model exactly the same cloud of the SW vs. height for this particular Rock type instead of the traditional way of using one trend function for each Rock Type. (Figure.7) One more QC step was done by taking off 3 wells from the conditional distribution as a blind test to QC how `s the estimator works. As we can see we have a very good correlation between the SW $\log$ and the estimated SW from the blind test using cloud transform. (Figure.8)

\section{Conclusions:}

Cloud Transform is a stochastic simulation technique which allows modeling Reservoir properties (especially $\mathrm{K}$ and SW) using a non-linear relationship to estimate the $\mathrm{K}$ from the PHI, and SW from the height parameter. Cloud transform has captures the reservoir heterogeneity.

Very Good estimate of Permeability from Porosity. Very good history match without using any permeability multipliers).

Could Transform results are Conditioned to well data. Cloud Transform takes fully into account the scatter between PHI and $\mathrm{K}$, or height and SW. The Cloud Transform can be done by Facies.

\section{ACKNOWLEDGMENTS:}

We would like to express our appreciation to the management and to UDR team at ADMA-OPCO for the opportunity to work on such an interesting and challenging project.

Sabah Karim, for his continuous support, Gary Mercado for providing the seismic interpretations and providing important evidence to aid modeling the faults, and Hamdan Manhali for his support in data gathering

\section{REFERENCES:}

Bashore, V M.; Araktingi U.G.; Levy M; Schweller U.G. Importance of a Geological Framework for Reservoir modeling and subsequent Fluid -Flow Predictions AAPG Computer application in geology., No.3; Jeffery M. Yarus and Richard L Chambers eds.; 1994.

Silverman B.W. Density Estimation for Statistics and Data Analysis. Chapman \& Hall 1986. 


\section{LIST OF FIGURES}

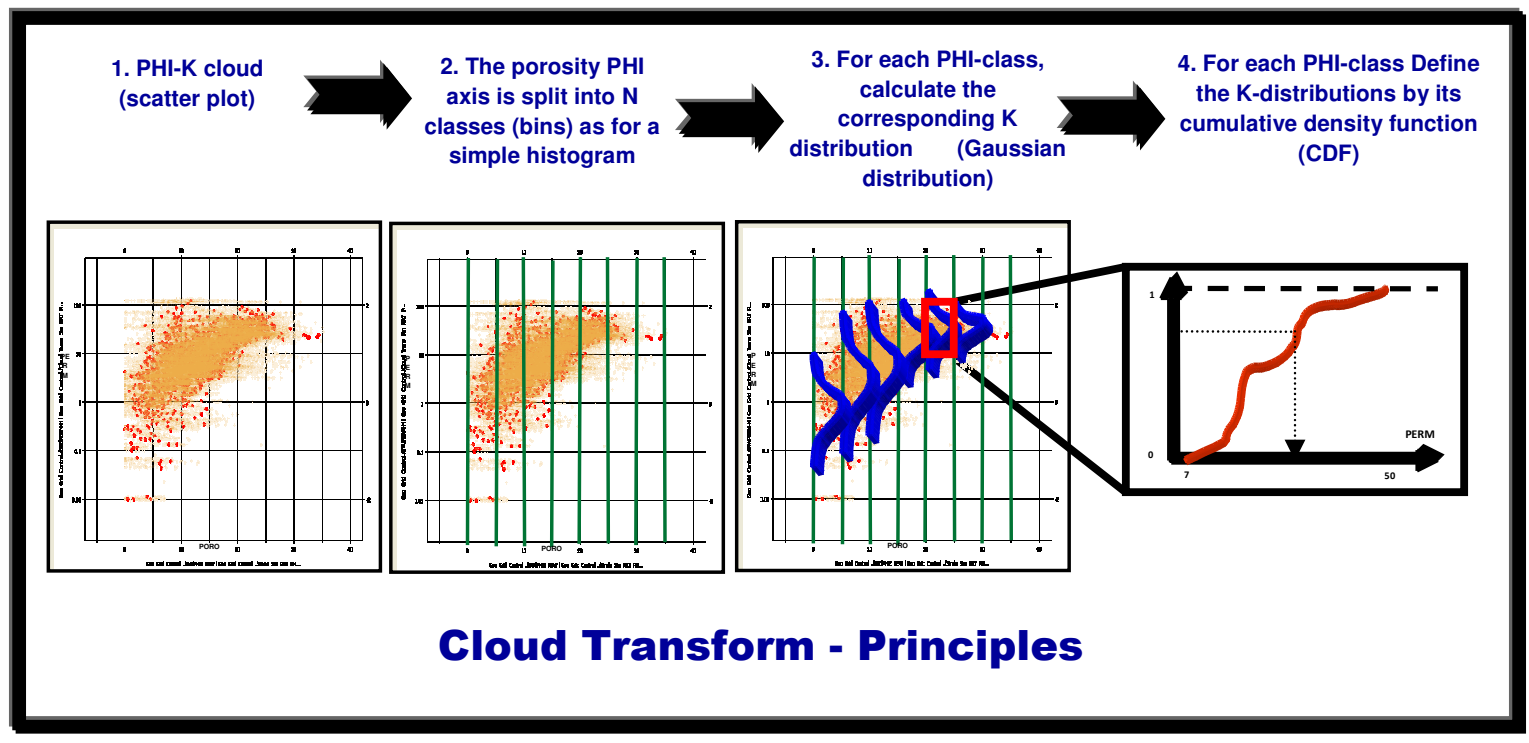

(Figure.1): Cloud Transform Principles

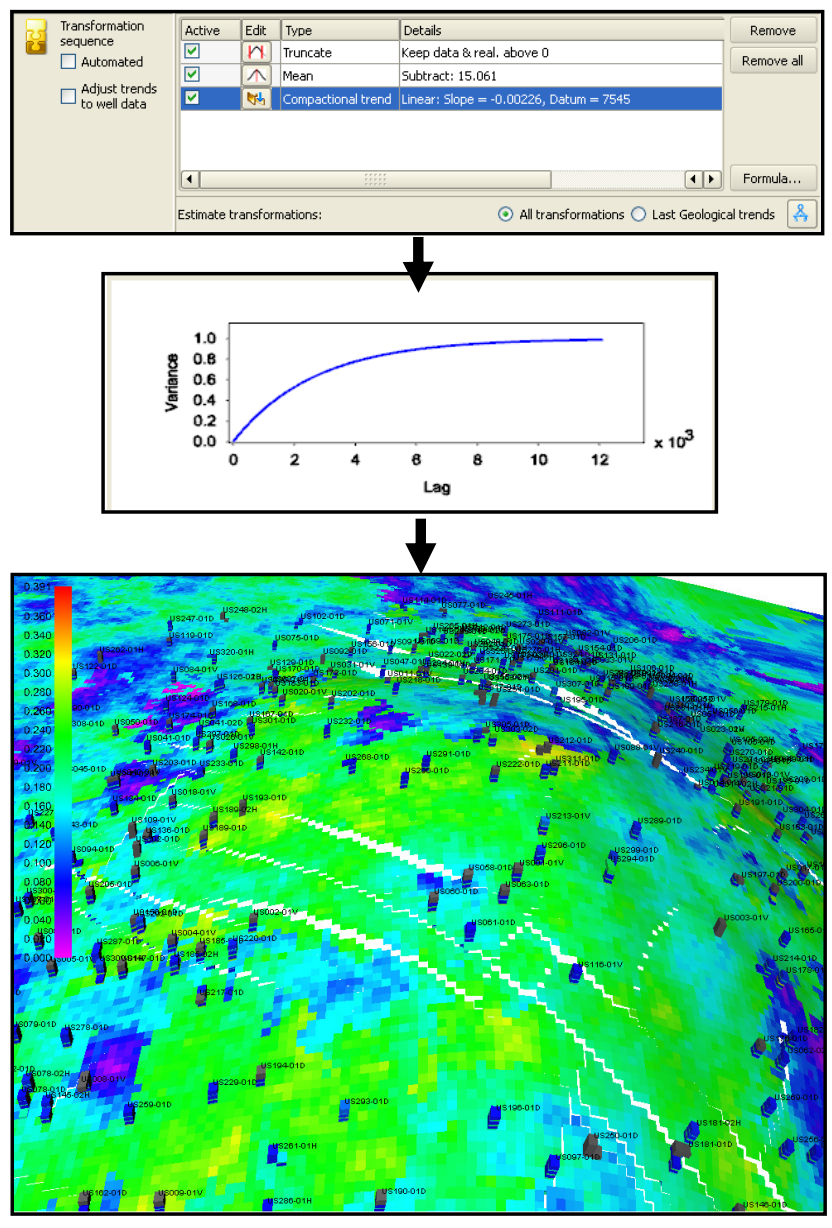




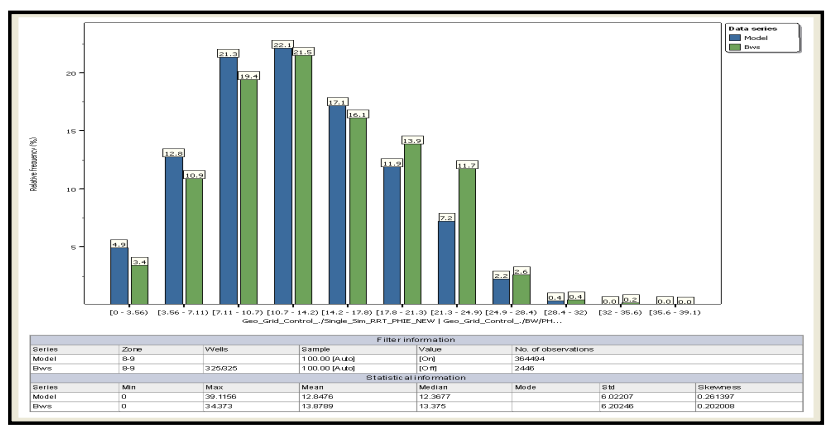

(Figure.2): Porosity modeling workflow and QC Histogram shows the modeling result (Blue) vs. well data (Green).

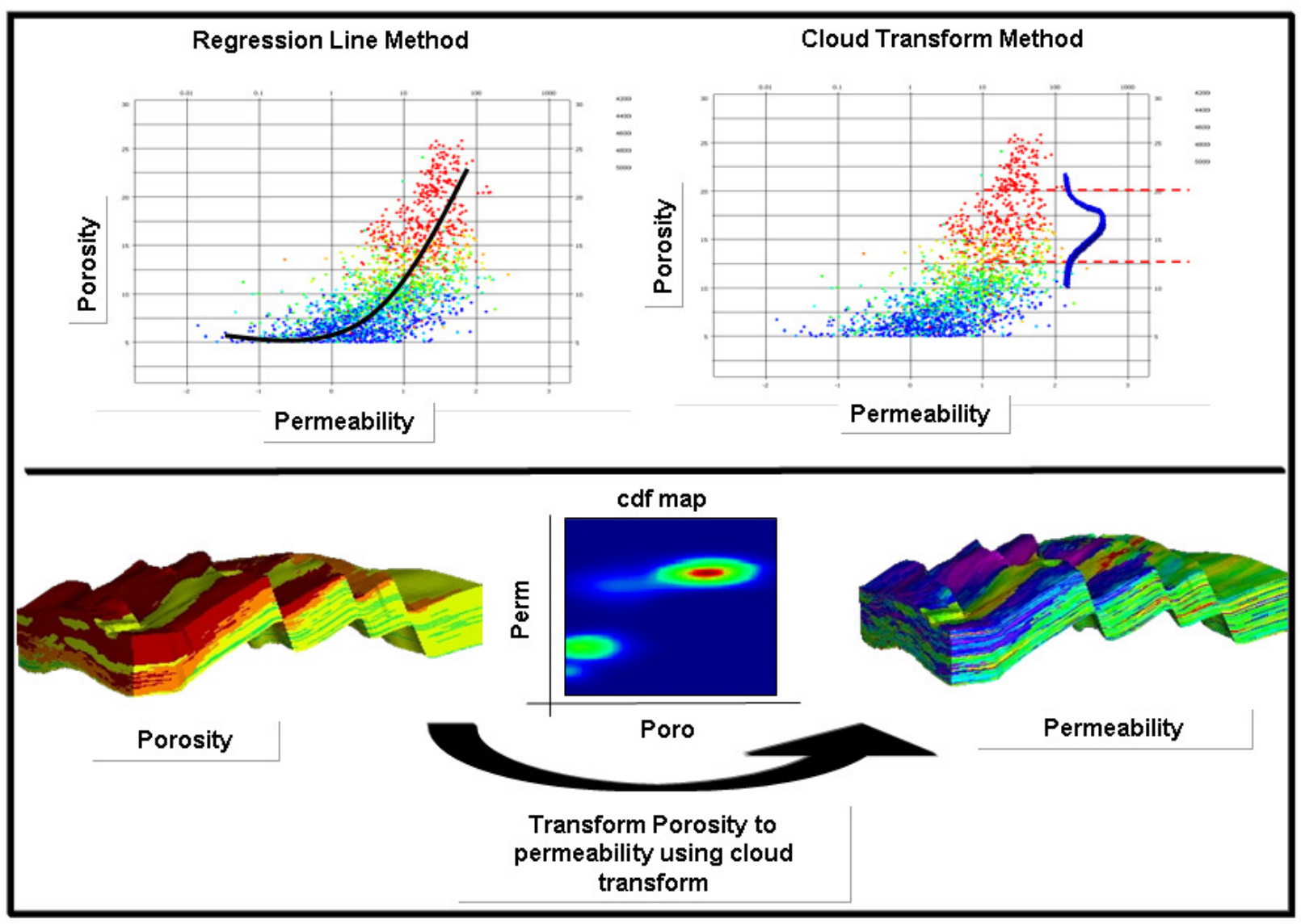

(Figure.3): Permeability modeling workflow using cloud transform. 


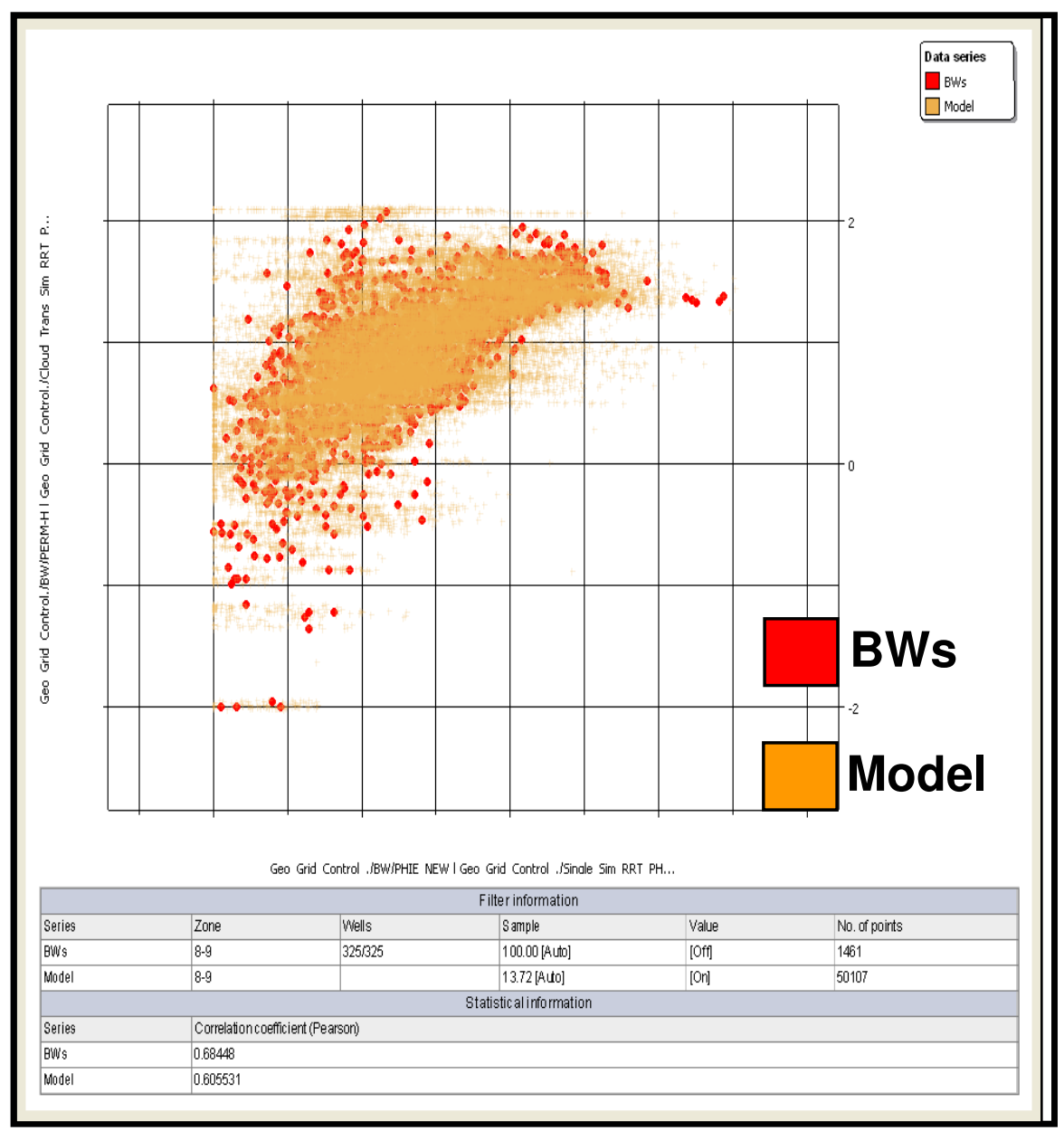

(Figure.4): Permeability model QC. The scatter plot shows the modeled permeability (Orange) vs. blocked wells and shows a perfect match. 


\section{Dynamic Model History \\ Production Performance from Two existing wells completed in the reservoir zone}

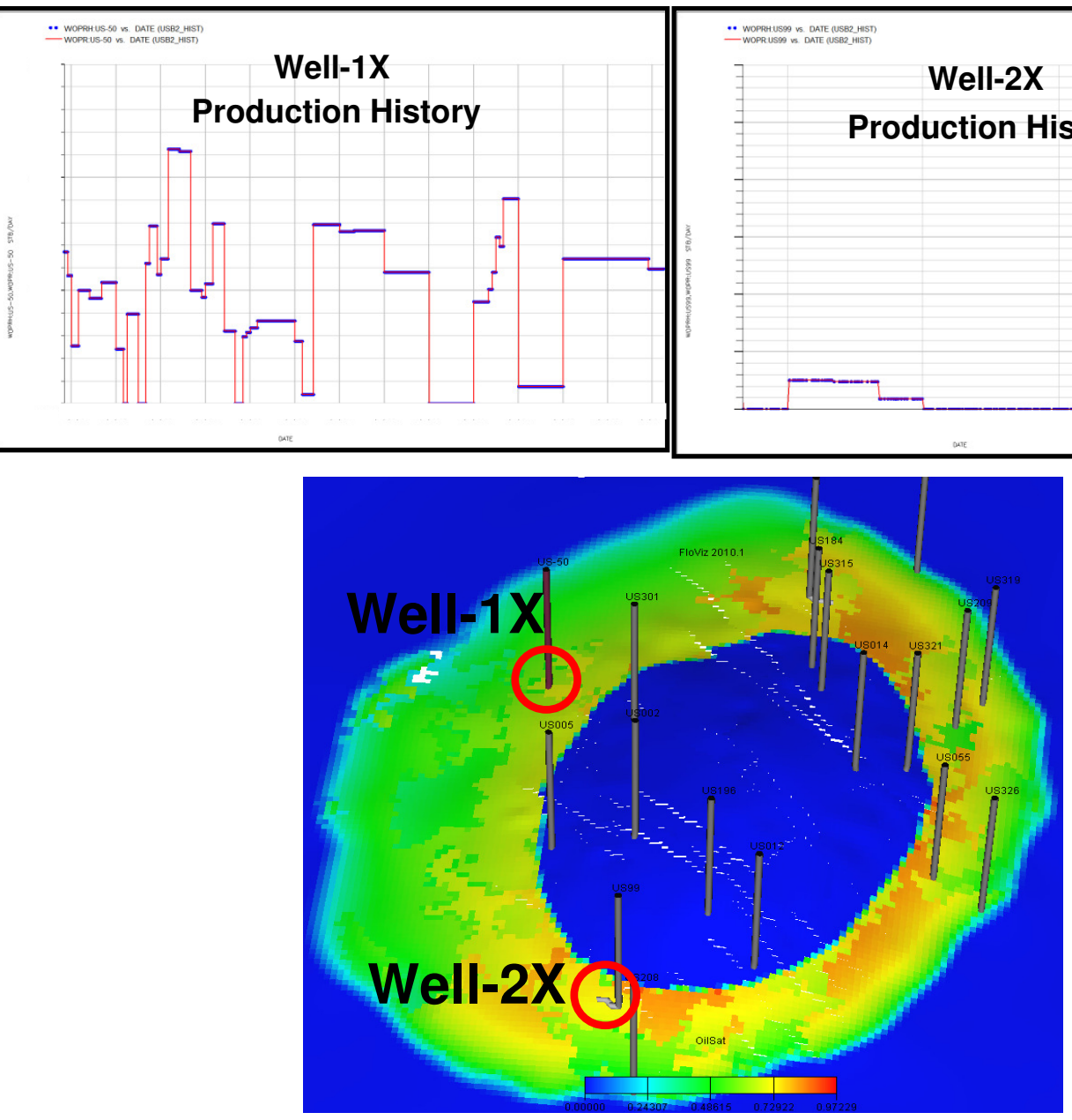

(Figure.5): Dynamic History match results show how the model perfectly matches the production history without a need to apply any permeability multipliers as usual. 


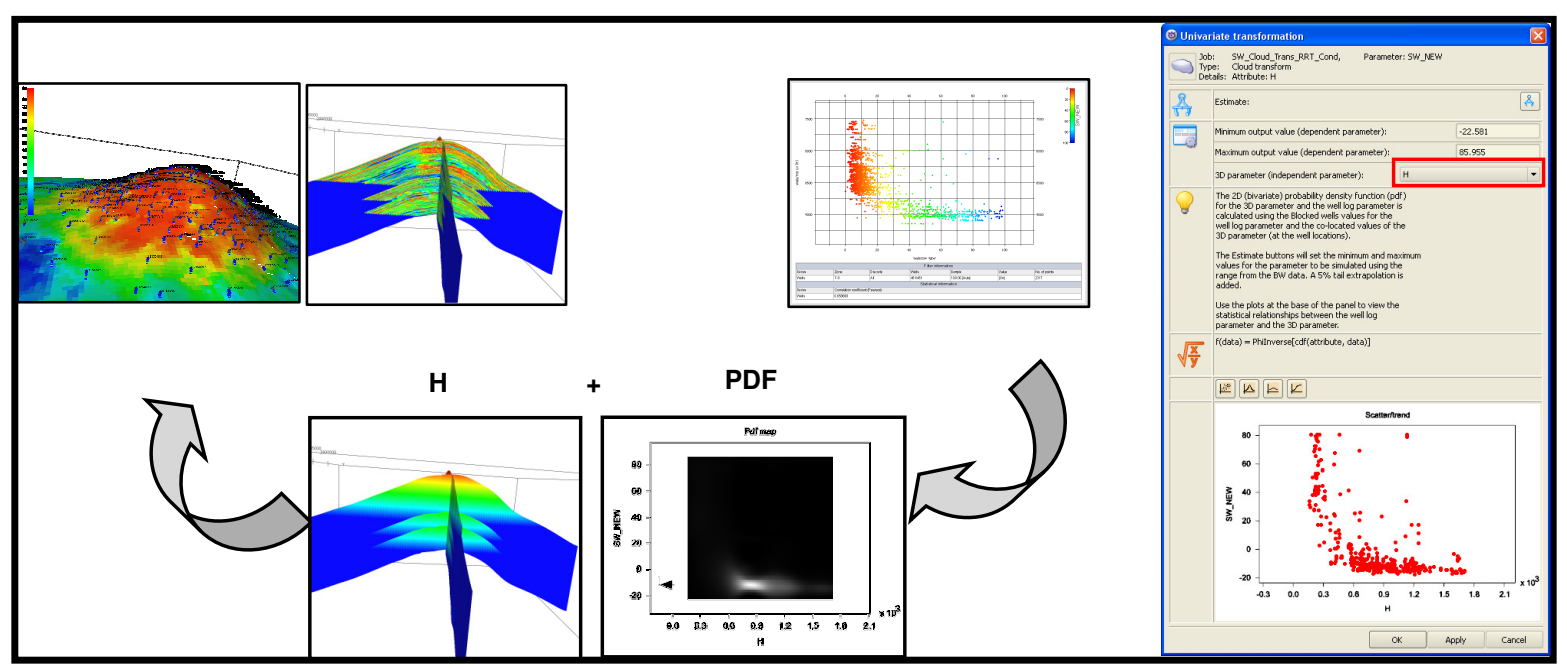

(Figure.6): Water saturation modeling workflow using cloud transform

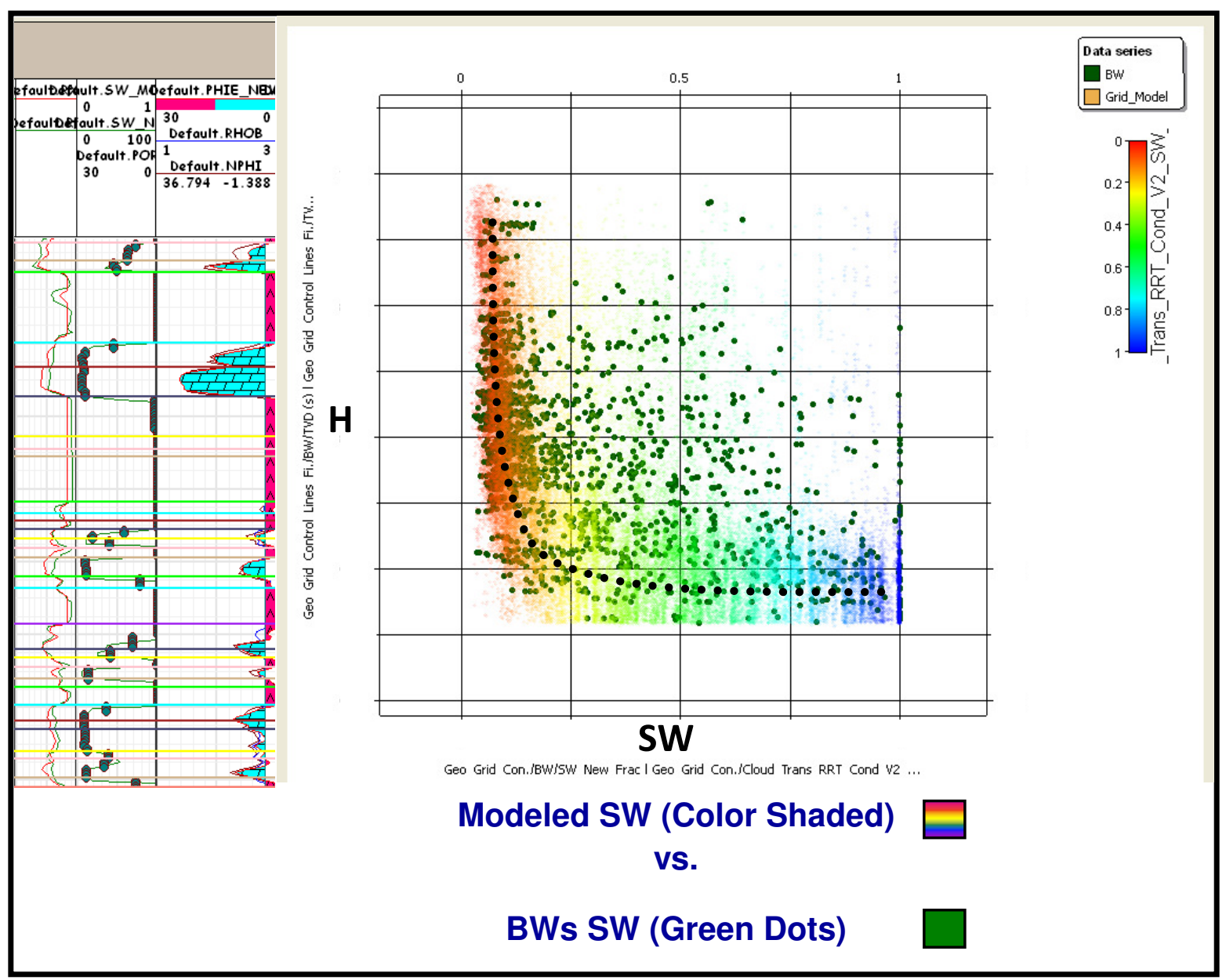

(Figure.7): Correlation between modeled SW (Color Shaded) Vs. BWs SW (Green Dots) shows how the model reproduced the same scatter plot of SW and capture the overall heterogeneity in the reservoir instead of the traditional way of using one trend function for each Rock Type(Black Dots). 


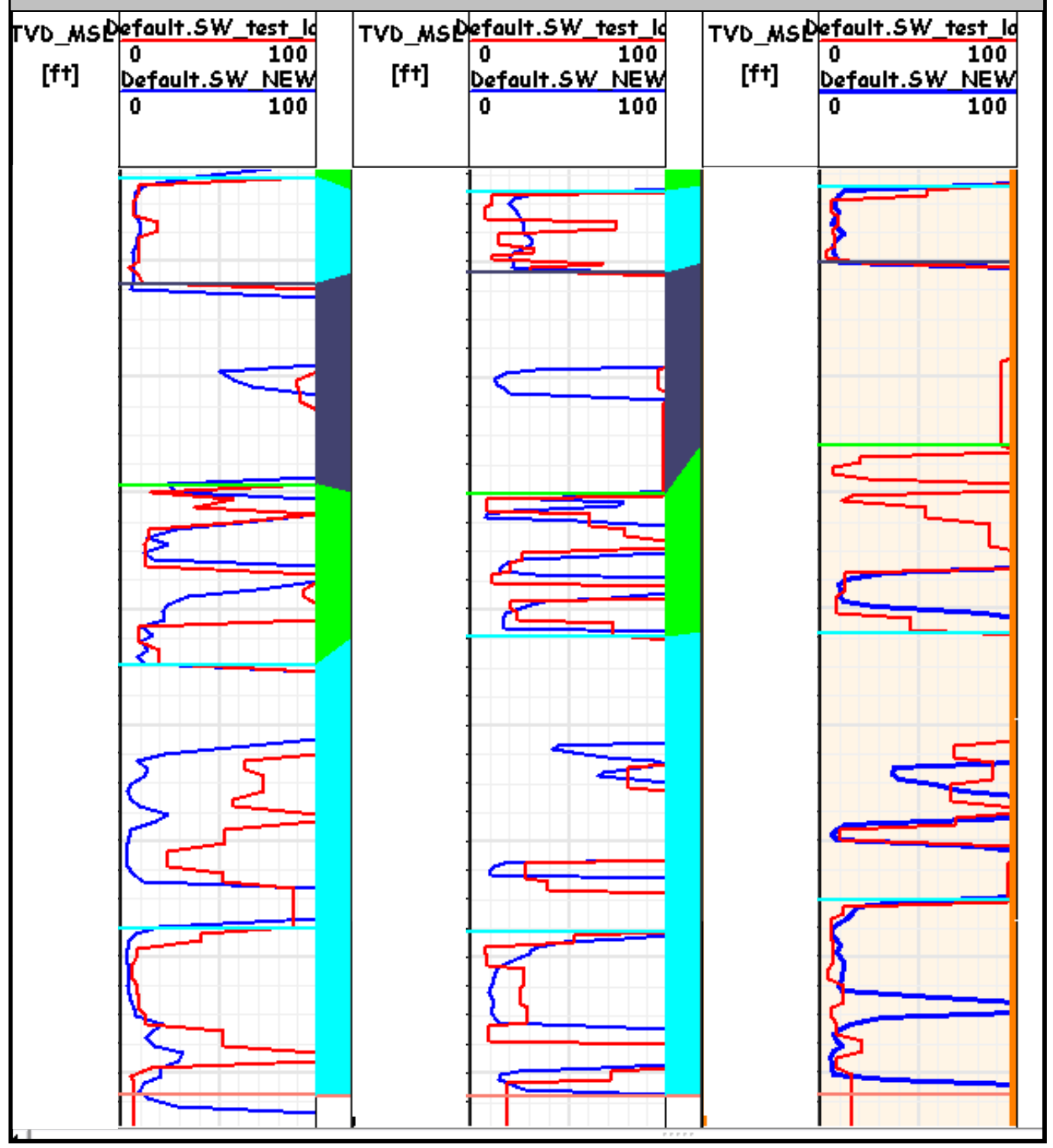

(Figure.8): Correlation between modeled SW from the blind test (Red Line) and the SW Log (Blue Line), We can see the perfect match between both. 